\title{
Mental health of healthcare workers during the COVID-19 outbreak: A rapid scoping review to inform provincial guidelines in South Africa
}

\author{
L J Robertson, ${ }^{1,2}$ MB BCh, FC Psych, MMed (Psychiatry); I Maposa, ${ }^{3}$ BSc, MSc (Statistics), PhD (Statistics); \\ H Somaroo, ${ }^{4,5}$ MB BCh, MsEpi, FCPHM (SA), MMed (Public Health Medicine); O Johnson, ${ }^{6,7}$ BSc, MBBS \\ ${ }^{1}$ Department of Psychiatry, School of Clinical Medicine, Faculty of Health Sciences, University of the Witwatersrand, Johannesburg, South Africa \\ ${ }^{2}$ Community Psychiatrist, Sedibeng District Health Services, Gauteng, South Africa \\ ${ }^{3}$ Division of Epidemiology and Biostatistics, School of Public Health, Faculty of Health Sciences, University of the Witwatersrand, Johannesburg, \\ South Africa \\ ${ }^{4}$ Department of Community Health, School of Public Health, Faculty of Health Sciences, University of the Witwatersrand, Johannesburg, \\ South Africa \\ ${ }^{5}$ Charlotte Maxeke Johannesburg Academic Hospital, Gauteng Department of Health, South Africa \\ ${ }^{6}$ Centre for Implementation Science, Health Services and Population Research Department, Institute of Psychiatry, Psychology and Neuroscience, \\ King's College London, UK \\ ${ }^{7}$ Centre for Health Policy, School of Public Health, Faculty of Health Sciences, University of the Witwatersrand, Johannesburg, South Africa
}

Corresponding author: L J Robertson (lesley.robertson@wits.ac.za)

COVID-19 is prevalent in sub-Saharan Africa. The healthcare response to the pandemic depends upon a mentally and physically healthy workforce. Infectious disease outbreaks cause high psychosocial stress among healthcare workers, which may impact negatively on workplace functioning. To understand which mental health conditions may occur and which interventions could be considered, we conducted a rapid scoping review. Using a 2018 systematic review as the starting point, PubMed, Cochrane, Web of Science and MEDLINE databases were searched for any type of evidence published in English between 2014 and 2020 on mental health of healthcare workers exposed to infectious disease outbreaks; 19 primary studies and 13 opinion pieces were included. Depression, anxiety, post-traumatic stress, and other mental health conditions were noted among healthcare workers exposed to COVID-19 and other outbreaks. Although no effectiveness studies were identified, certain proposed interventions may be implemented by healthcare leaders. Further research is recommended.

S Afr Med J.2020:110(10):1010-1019. https://doi.org/10.7196/SAMJ.2020.v110i10.15022

COVID-19, caused by the novel severe acute respiratory syndrome coronavirus 2 (SARS-CoV-2), is highly transmissible, with a high mortality in vulnerable individuals and no known disease-specific treatment or vaccine. ${ }^{[1]}$ On 11 March 2020, the World Health Organization (WHO) declared the COVID-19 outbreak to be a pandemic, making global recommendations to reduce transmission, prepare and be ready. ${ }^{[2]}$ As anticipated, it continued to spread and is now prevalent in South Africa (SA) and other African countries, ${ }^{[1]}$ putting healthcare systems under severe pressure.

Healthcare workers (HCWs) are the most important resource in both the COVID-19 response and in maintaining essential services. ${ }^{[3]}$ HCW motivation and empathy are critical to effective and compassionate healthcare. Mental health conditions may compromise work performance and increase risk of burnout, absenteeism and resignations. ${ }^{[4,5]}$ Fatigue, emotional exhaustion or poor concentration are likely to increase clinical error, including breaches in infection control, impacting on patient care and staff infections. Protecting the mental health and wellbeing of HCWs is therefore of paramount importance during an infectious disease outbreak.

Such outbreaks cause high levels of psychosocial stress, ${ }^{[6]}$ related to uncertainty regarding risk of infection and prognosis, loss of loved ones, and the social impact of measures instituted to contain the spread of disease. Being at the frontline in disease detection and management, HCWs are vulnerable, exposed to infection, high workloads and difficult working conditions. Resource constraints, including shortages of personal protective equipment (PPE), limit individual capacity to provide appropriate patient care and heighten the mental health risk. Being quarantined appears to convey particular stress. ${ }^{[7]}$

The Gauteng Province Department of Health's research and evaluation workstream therefore requested evidence-based recommendations regarding the potential impact of the COVID-19 pandemic on the mental health of HCWs and their protection. This article describes the process behind, and builds upon, a presentation made to the senior leadership of the Gauteng Department of Health's COVID-19 response team in April 2020, for which we sought to answer two questions: (i) what may be expected regarding the psychological impact of the COVID-19 outbreak on HCWs; and (ii) what interventions could be considered in order to protect and support the mental health and wellbeing of HCWs during the crisis.

\section{Methods}

We conducted a rapid scoping review of published literature on mental health of HCWs exposed to infectious disease outbreaks.

\section{Literature search and study selection}

To gain an understanding of the topic, we conducted a preliminary search and screen of the PubMed and Cochrane databases on 7 and 8 April 2020, respectively, using terms (healthcare workers OR medical doctors OR nurses OR community health workers) AND 
(mental health OR psychiatry) AND (COVID-19 OR coronavirus OR pandemic OR outbreak), for any publications in English. While the PubMed search yielded 595 records, no records were retrieved from Cochrane. We also examined reference lists of guidelines from various organisations and met with a local team of mental health specialists offering volunteer support to HCWs during COVID-19 ('Mental health care for COVID-19 healthcare workers' (https:// covidcaregauteng.co.za/), accessed 14 May 2020), who shared the literature they had sourced to inform their programme.

We decided to use a systematic review by Brooks et al. $(2018)^{[8]}$ as the starting point for our review, because of its relevance to our review questions and its satisfactory quality. Brooks et al. performed a thematic analysis, exploring social and occupational factors affecting the psychological wellbeing of HCWs exposed to the severe acute respiratory syndrome coronavirus 2002 (SARS-CoV-1) pandemic. In terms of quality, they conducted a comprehensive literature search, selected studies in duplicate according to clearly defined inclusion criteria, and provided a detailed list of included studies with appropriate quality appraisal.

Based on our initial reading of the literature, we developed a set of inclusion and exclusion criteria for our review, as set out in Table 1. We also developed an iterative search strategy for articles published between 2014 and 2020. The date limits were chosen to overlap with the search by Brooks et al., which was conducted in 2015. Using the search string developed by Brooks et al. with the addition of terms COVID*, coronavirus and SARS-CoV-2 (Appendix 1, http:// samj.org.za/public/sup/15022-1.pdf), author OJ searched the Web of Science and MEDLINE databases on 17 April 2020. The records retrieved were downloaded to Excel, version 2007 (Microsoft, USA), where duplicates were removed. Author LJR evaluated the titles, abstracts and full text for inclusion, discussing uncertainty with the other three authors.

\section{Data extraction and synthesis}

Data were extracted by LJR onto prepared tables in Microsoft Word, version 2007 (Microsoft, USA). For primary studies, the year of publication, disease outbreak, country, study design, participant information, outcomes, tools used and key findings were extracted. For opinion pieces, the year of publication, disease outbreak, country and key content were documented.

To synthesise the data, we conducted a descriptive analysis using categories based on our review questions. We developed four categories: the types and prevalence of mental health conditions identified; related workplace consequences; risk and protective factors; and possible interventions. Contingent with scoping review guidelines, ${ }^{[9]}$ we did not conduct a quality appraisal of included articles.

\section{Results}

Of the 31 articles identified for inclusion in addition to Brooks et al. ${ }^{[8]} 18$ reported on primary studies and 13 were opinion pieces. The selection process is presented in Fig. 1, and the included articles and extracted data are tabulated in Appendix 2 (primary studies, http://samj.org.za/public/sup/15022-2.pdf) and Appendix 3 (opinion pieces, http://samj.org.za/public/sup/15022-3.pdf). Infectious disease outbreaks covered were COVID-19 (12 studies and 10 opinion pieces), the Middle Eastern respiratory syndrome coronavirus (MERS-CoV) outbreak of 2012 (4 studies), the 2014 Ebola outbreak (2 studies and

\begin{tabular}{|c|c|c|}
\hline Study characteristic & Inclusion criteria & Exclusion criteria \\
\hline Population & $\begin{array}{l}\text { Healthcare workers employed within the healthcare } \\
\text { system (e.g. nurses, medical doctors, allied health } \\
\text { professionals, community health workers, healthcare } \\
\text { support staff) }\end{array}$ & $\begin{array}{l}\text { - Health professional students (e.g. medical or } \\
\text { nursing students) } \\
\text { - Workers outside the healthcare system (e.g. } \\
\text { municipal workers, social service workers) } \\
\text { - Patients } \\
\text { - Family or lay caregivers } \\
\text { - General public }\end{array}$ \\
\hline Concept & $\begin{array}{l}\text { Any mental health condition, including medically } \\
\text { diagnosable mental disorders and non-diagnosable } \\
\text { conditions such as stress, moral injury, emotions } \\
\text { - Factors related to mental health conditions, e.g. types, } \\
\text { prevalence, and severity; risk and protective factors; } \\
\text { interventions }\end{array}$ & $\begin{array}{l}\text { - Non-mental health conditions, e.g. social or } \\
\text { physical health conditions }\end{array}$ \\
\hline Context & $\begin{array}{l}\text { Exposure to an infectious disease outbreak, whether } \\
\text { frontline or non-frontline, at any healthcare service } \\
\text { level }\end{array}$ & $\begin{array}{l}\text { - Disasters or emergencies that are not infectious } \\
\text { disease outbreaks } \\
\text { - Normal working conditions }\end{array}$ \\
\hline Publication & $\begin{array}{l}\text { - Published in an academic journal } \\
\text { - English }\end{array}$ & $\begin{array}{l}\text { - Non-journal publications, e.g. website } \\
\text { publications, guidelines or recommendations } \\
\text { issued by an organisation, flyers, and blogs } \\
\text { - Grey literature } \\
\text { - Publications in languages other than English }\end{array}$ \\
\hline Types of evidence & $\begin{array}{l}\text { - Any, including primary studies and opinion pieces } \\
\text { - For primary studies, we included any exploratory } \\
\text { study, even brief interviews or rapid needs } \\
\text { assessments, as long as the methods were described } \\
\text { - For opinion pieces, we included any published } \\
\text { opinion article, such as guidelines, narrative reviews, } \\
\text { editorials, commentaries or personal views, and } \\
\text { correspondence }\end{array}$ & - Not applicable \\
\hline
\end{tabular}




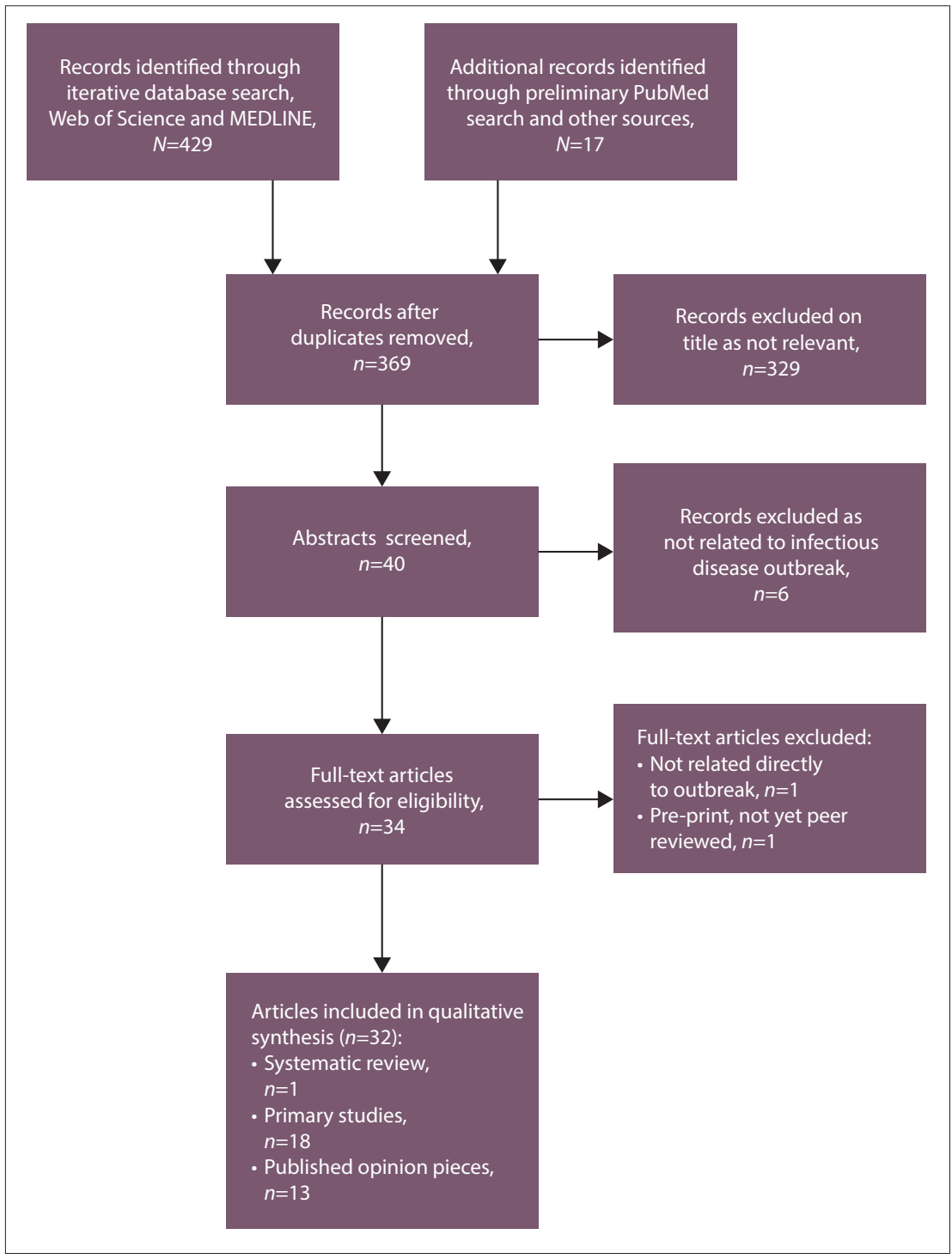

Fig. 1. Flow chart of article screening and selection.

3 opinion pieces), and SARS-CoV-1 (the systematic review of 22 studies by Brooks et al. ${ }^{[8]}$ ). While the opinion pieces referring to COVID-19 drew on literature from previous outbreaks as well as experience of the current pandemic, those referring to the Ebola outbreak drew only on experience of that epidemic.

All primary studies were cross-sectional in design; 13 used self-report questionnaires, 4 were interview based, and 1 used mixed methods. Response rates were reported in 11 studies and ranged from $19.9 \%$ to $100 \%$. Sample sizes varied from 30 to 2299 participants for questionnaire-based surveys, and from 10 to 69 participants for exploratory interviews. Female respondents predominated, accounting for $>75 \%$ of participants in 11 studies. Opinion pieces comprised 6 commentaries or personal views, 4 letters, an editorial, a narrative review, and a description of a pilot project in
West Africa. All the included articles related to hospital-based HCWs; none referred to primary healthcare or community-based services.

\section{Types and prevalence of mental health conditions}

Table 2 summarises the prevalence rates available from questionnaire-based studies for depression, ${ }^{[10-14]}$ anxiety, ${ }^{[11-14]}$ post-traumatic stress (PTS), ${ }^{[11,13,15,16]}$ insomnia, ${ }^{[10,11,14]}$ mental disturbance (depression, anxiety, PTS and insomnia grouped together), ${ }^{[17]}$ somatisation, ${ }^{[10,14,17]}$ obsessive-compulsive symptoms, ${ }^{[14]}$ burnout, ${ }^{[10]}$ stress $^{[13,18]}$ and fear. ${ }^{[12]}$ Medical HCWs comprise nurses, doctors and clinical assistants, while nonmedical HCWs are a nonspecific group of allied health professionals and support staff. All studies assessed symptomatology rather than diagnosis, using a variety of screening tools, and some included conditions that are not medically diagnosable, notably burnout, stress and fear. The severity but not the prevalence of another non-diagnosable condition, vicarious traumatisation, was evaluated in one study in Hubei province during COVID-19. ${ }^{[19]}$ Vicarious traumatisation refers to psychological distress arising from sympathy with trauma victims. Using a questionnaire developed previously in China after a natural disaster, Li et al. $(2020)^{[19]}$ found greater severity among non-frontline nurses and among the general public compared with frontline nurses.

Moral injury (a reflection of dissonance between clinical practice and personal values) was not assessed in any primary studies. However, it was discussed by Greenberg et al. $(2020)^{[20]}$ and Walton et al. $(2020)^{[21]}$ with regard to COVID-19 exposure, and by Ulrich (2014) ${ }^{[22]}$ regarding distress among West African HCWs exposed to the 2014 Ebola epidemic.

Similarly, anger was not evaluated in any of the primary studies, although angry or aggressive emotional reactions were noted in several opinion pieces. ${ }^{[20,21,23-25]}$ In addition, Brooks et al.$^{[8]}$ found that being quarantined and poor organisational support each predicted high levels of anger among HCWs.

We found no assessment of substance use, psychotic illness or suicidal behaviour. However, Brooks et al. ${ }^{[8]}$ found excessive alcohol intake to be associated with working in a high-risk environment and with being quarantined. They also mentioned psychoticism in relation to the SARS-CoV-1 outbreak, and Ho et al. $(2020)^{[23]}$ warn of it as a possible occurrence during COVID-19. Likewise, Ho et al. warn of suicidality, which they suggest may be related to self-blame.

Long-term mental health effects were also not evaluated, except by one study included in the review by Brooks et al. ${ }^{[8]}$ In a 3-year follow-up of HCWs exposed to SARS-CoV-1 in China, Wu et al. (2009) ${ }^{[26]}$ found that $10 \%$ had persistent PTS symptoms, and that altruistic acceptance during the outbreak was a protective factor.

\section{Work-related consequences}

Only one study evaluated a work-related outcome associated with mental health. Among nurses exposed to the MERS-CoV outbreak in South Korea, Jung et al. (2019) ${ }^{[15]}$ found a greater intention to resign to be associated with increased severity of PTS.

However, a range of work-related outcomes were commented on by Brooks et al. ${ }^{[8]}$ and other authors. ${ }^{[1,17,20,21,25,27-31]}$ Fatigue, irritability, and interpersonal conflict with colleagues 







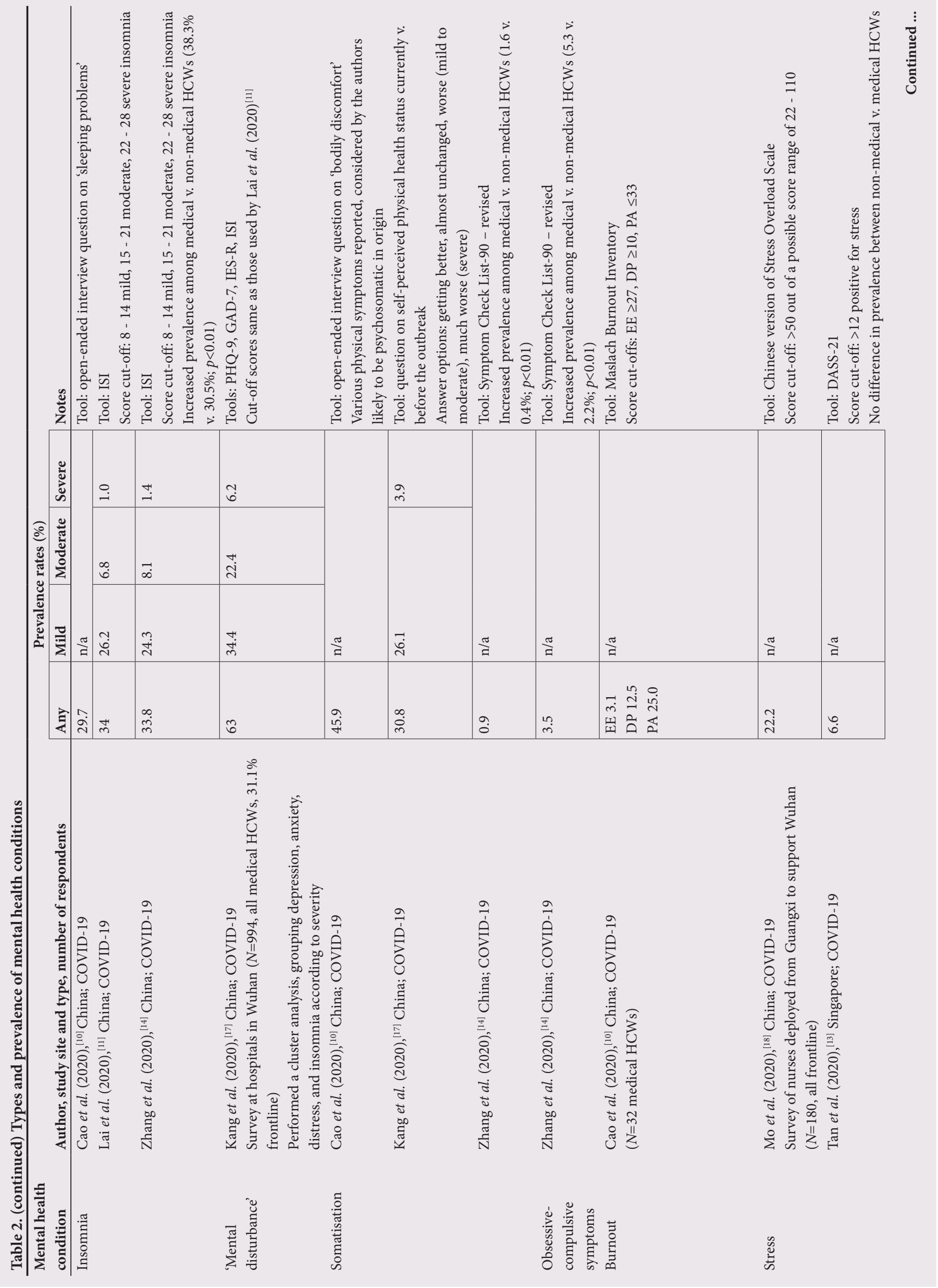




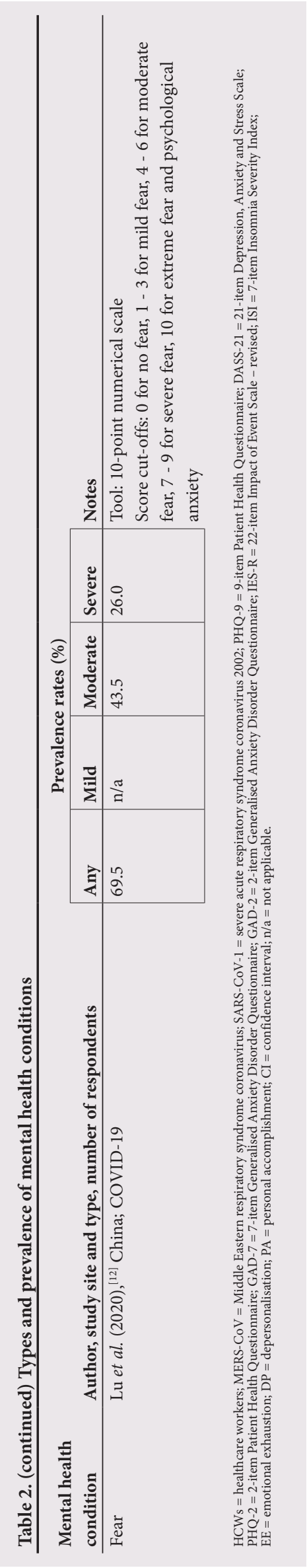

were described in association with stress. ${ }^{[14,19,21,31]}$ Caution was expressed regarding presenteeism related to burnout and poor mental health. ${ }^{[20]}$ Medical error was warned of by authors from Iran, ${ }^{[30]}$ and reluctance to work was noted in three articles. ${ }^{[8,21,28]}$ Finally, the possibility of legal implications arising from lapses in 'duty of care' was raised by Greenberg et al. (2015), ${ }^{[27]}$ writing from their experience of supporting HCWs during the Ebola virus outbreak.

\section{Risk and protective factors}

Workplace, social and individual HCW risk and protective factors identified by Brooks et $a .^{[8]}$ and by questionnaire-based ${ }^{[11-19,32-34]}$ and interview-based ${ }^{[29,31,35]}$ studies are summarised in Table 3, with more detail per study provided in Appendix 2. While being a frontline worker was identified as a prominent risk factor, being non-frontline or a non-medical HCW were also associated with high risk of mental disturbance. ${ }^{[13,14,19]}$ Tan et al. ${ }^{[13]}$ speculated that poorer access to psychological support, less skills training, and less medical knowledge regarding the outbreak may have caused greater mental health risk to non-medical compared with medical HCWs. They attributed the lower rate of PTS found during COVID-19 than during SARS-CoV-1 to improved psychological preparedness and more stringent infection control in the current pandemic.

Inadequate resources to manage the caseload were a notable source of stress in several studies, ${ }^{[12,29,31,35]}$ and working at a secondaryrather than a tertiary-level hospital was found to be predictive of depression, anxiety and insomnia by Lai et al.(2020 ${ }^{[11]}$ A qualitative study among HCWs in Sierra Leone ${ }^{[29]}$ during the Ebola outbreak documented high stress levels related to poor health system infrastructure, undermining psychological preparedness and necessitating strong individual coping strategies.

Four studies explored relationships between various risk and protective factors. In Wuhan, Kang et al. (2020) ${ }^{[17]}$ found that increased COVID-19 exposure predicted worse physical health perception through its negative effect on mental health disturbance. Accessing mental healthcare services moderated this relationship through improved mental health. Xiao et al. $(2020)^{[32]}$ found that social support indirectly improved sleep quality by reducing anxiety and stress and strengthening self-efficacy. Jung et al. ${ }^{[15]}$ found that stronger supervisor support appeared to buffer the effect of PTS on nurses' intention to resign. Park et al. (2018) ${ }^{[34]}$ found that the mental health effects of stigma and of innate resilience were partially mediated by stress.

\section{Possible interventions}

No effectiveness studies were identified in our literature search. Rather, interventions were recommended according to identified needs, coping strategies, risk and protective factors, as well as from personal experience. While some articles ${ }^{[11,12,16,17,19,30]}$ prioritised early recognition and individual psychological support, others $^{[8,10,15,18,20,21,23-25,27,31,33,35-38]}$ placed emphasis on organisational interventions to support HCWs. Four studies focusing exclusively on nurses $^{[15,18,34,39]}$ made recommendations to mitigate work stress, enhance innate resilience and provide team leader support. To promote institutional resilience, Wu et al. (2020) $)^{[24]}$ suggested that staff support be included as a priority logistical process, alongside infection control and supply chain management.

Two articles categorised interventions according to whether they were implemented before, during or after the peak of an outbreak. ${ }^{[20,37]}$ A narrative review ${ }^{[21]}$ sorted interventions according to role-players, with differing responsibilities for health institutions, team leaders and individual HCWs, and one article considered them in terms of primary, secondary and tertiary disease prevention strategies. ${ }^{[27]}$ We have grouped interventions according to management phases, using the WHO occupational health and safety nomenclature. ${ }^{[3]}$

\section{Pre-deployment}

For the preparatory phase, Greenberg et al. ${ }^{20]}$ advised that anticipated duties and potential traumatic situations be explained clearly and accurately to HCWs, and that a cohesive team spirit be fostered to facilitate positive coping mechanisms. Chen et al. $(2020)^{[31]}$ highlighted the need for pre-job skills training in the management of distressed and unco-operative patients, as well as infection control measures and PPE. Describing a project used during the Ebola outbreak, Schreiber et al. (2019) ${ }^{[37]}$ recommended that HCWs be trained in self-monitoring for symptoms of distress or mental illness, internal coping skills, and how to access external psychological resources. Several articles ${ }^{[10,23-25,27]}$ recommended that organisations establish peer support networks and psychological support systems. Psychological preparation of managers and team leaders, empowering them to provide the quality of leadership required during the outbreak, was emphasised by Wu et al. ${ }^{24}$

\section{During deployment}

As the outbreak peaks and work demands escalate, visible, decisive leadership, responsive to the concerns of HCWs, with clear, open communication, is recommended. ${ }^{[21,24,36]}$ Four articles $^{[10,31,35,36]}$ demonstrated responsive leadership, as they reported on rapid needs assessments and the corresponding actions taken by healthcare institutions. While 
Table 3. Risk and protective factors for mental health conditions

\begin{tabular}{|c|c|c|}
\hline & Risk factors & Protective factors \\
\hline Workplace & $\begin{array}{l}\text { - High perceived risk of contagion; hazardous environments; } \\
\text { inadequate PPE; unclear infection control measures } \\
\text { - Inadequate training in infectious disease outbreaks; not } \\
\text { feeling competent } \\
\text { - Incapacity to meet patient needs; difficulty managing } \\
\text { unco-operative or anxious patients; witnessing suffering } \\
\text { of patients and their relatives; caring for colleagues when } \\
\text { infected } \\
\text { - Poor control over work conditions; inadequate resources } \\
\text { to do one's job properly; heavy caseload; involuntary } \\
\text { deployment; long working hours } \\
\text { - Weak organisational structure; poor communication and } \\
\text { psychological support; unclear job instructions; sense of } \\
\text { being blamed; HCW concerns not reaching management }\end{array}$ & $\begin{array}{l}\text { - Adequate infection control measures; clear instructions } \\
\text { regarding use and maintenance of PPE } \\
\text { - High degree of knowledge; specialised training; accurate } \\
\text { information regarding risk and illness } \\
\text { - Support from supervisors, trained personnel, and peers; } \\
\text { open discussions about difficult situations } \\
\text { - Flexibility in workplace: designated rest periods; } \\
\text { rotational shifts; shorter working hours } \\
\text { - Two-way dialogue between management/supervisors } \\
\text { and HCWs; opportunity for HCWs to provide feedback } \\
\text { and sense of being heard }\end{array}$ \\
\hline Social & $\begin{array}{l}\text { - Stigmatisation and social isolation related to COVID-19 } \\
\text { exposure } \\
\text { - Inability to maintain home life } \\
\text { - Worries over family responsibilities } \\
\text { - Poor family or social support }\end{array}$ & $\begin{array}{l}\text { - Social support from families and the community; } \\
\text { communication with families } \\
\text { - Media information restrained and accurate } \\
\text { - Availability of self-help material and psychological } \\
\text { support }\end{array}$ \\
\hline $\begin{array}{l}\text { Individual } \\
\mathrm{HCW}\end{array}$ & $\begin{array}{l}\text { - Being front-line in diagnosis or care of COVID-19 } \\
\text { patients or being non-medical or support staff } \\
\text { - Being a nurse } \\
\text { - Being female, single, a parent, and/or an only child } \\
\text { - Having been quarantined } \\
\text { - Having a medical condition } \\
\text { - Past experience of trauma or loss } \\
\text { - Maladaptive coping skills }\end{array}$ & $\begin{array}{l}\text { - Innate resilience; sense of purpose; altruistic acceptance } \\
\text { - Positive coping strategies to manage isolation, personal } \\
\text { stress, work/life balance } \\
\text { - High sense of self-efficacy and competence }\end{array}$ \\
\hline
\end{tabular}

appropriate PPE and infection control measures were critical, addressing basic needs, including sleep, regular meals, rest periods and human connection, was also vital. In China, one institution adjusted working hours, with short 4 - 6-hour shifts and regular time off, ${ }^{[10]}$ whereas another provided a quiet rest area, food, systems to improve communication with families, continued skills training, and daily opportunities to discuss difficulties experienced at work. ${ }^{[31]}$ In the USA, regular, accurate updates on the outbreak were provided, as well as childcare facilities and transport to facilitate HCWs getting to work, accommodation if quarantine was warranted, and support regarding home stressors. ${ }^{[35,36]}$ The needs identified through these assessments were consistent with findings from in-depth individual interviews among nurses in Wuhan, ${ }^{[39]}$ in that personal health, safety, social relatedness, and reliable, scientific information predominated.

Other recommendations included strong supervisory and peer support, shared clinical decision-making, sharing of emotions, early recognition of distress, provision of psychological first aid, and encouragement of appropriate help-seeking. ${ }^{[21,24,27,29,36,38]}$ While singlesession psychological debriefing is considered potentially harmful, regular sessions in which difficult clinical decisions and situations are discussed with supervisors are recommended ${ }^{[20,21,31,37]}$ Three articles recommended active monitoring for mental health symptoms, with timely access to psychological interventions. ${ }^{[23,28,30]}$ In Wuhan, Kang et al. ${ }^{[17]}$ found that HCWs who accessed psychological support expressed benefit from doing so. Those with mild to moderate mental disturbance were more likely to use self-help or online psychological materials, whereas those with more severe symptoms sought counselling or psychotherapy.

\section{Post-deployment}

Integral to building institutional resilience is that HCWs and the health system are fit to continue serving the population once the pandemic has resolved. ${ }^{[24,38]}$ In an editorial, Kang et al. $(2020)^{[25]}$ emphasised the need to learn and improve the quality of future management of public health emergencies. Writing on moral injury, Greenberg et al..$^{[20]}$ recommend a period of reflection as a team as the outbreak subsides, with a focus on finding meaning and growth from the experience.

\section{Discussion}

In this review, we found evidence from cross-sectional exploratory studies of mental health conditions among hospital-based frontline and non-frontline HCWs during infectious disease outbreaks, as well as of risk and protective factors. We found published opinion pieces that recommended various protective interventions. However, we found no information specific to primary care or community-based healthcare services.

\section{Mental health conditions}

Several factors regarding the survey findings make it difficult to anticipate the prevalence or severity of mental health conditions among HCWs during the COVID-19 response in SA. Firstly, a wide range in prevalence of various symptoms was found. Rates of any depression, anxiety and PTS ranged from $8.9 \%$ to $50.4 \%$, $10.4 \%$ to $44.6 \%$ and $32 \%$ to $71.5 \%$, respectively. Differences in study population (e.g. proportions of frontline, medical or non-medical HCWs), study sites and screening tools may account for some of the variation in prevalence. However, as there was no comparison with 
mental health of HCWs during normal working conditions, it is not possible to gauge whether prevalence or severity were worse than usual for the individual study sites.

While there is a paucity of data on mental health of HCWs in Africa, ${ }^{[5]}$ understanding the global findings during infectious disease outbreaks against the background of those in Africa during normal conditions should help inform local planning. In SA during 2010, among 132 primary care doctors in Western Cape Province, ${ }^{[40]}$ 27\% reported moderate depression and 3\% severe depression. Among 67 doctors in North-West Province, ${ }^{[41]} 51 \%$ were found to be stressed and $27 \%$ highly stressed. More data are available regarding burnout, ${ }^{[42]}$ a result of 'chronic workplace stress that has not been successfully managed, according to the International Classification of Diseases. ${ }^{[43]}$ In their systematic review of burnout among HCWs in sub-Saharan Africa, Dubale et al. (2019) ${ }^{[42]}$ found rates of $62 \%$ in Malawi, 91\% in Ethiopia and 95\% in Kenya. In SA, they found rates of $46 \%$ among nurses at national referral hospitals, $78 \%$ among junior doctors, and $81 \%$ in a small sample of rural doctors. They also noted high rates of depression and anxiety among HCWs in some of the studies included in their review.

Secondly, whether the rates reflect incident morbidity in an individual, precipitated by stressful circumstances, or total morbidity, inclusive of pre-existing conditions, was not elucidated in any study. However, a recently conducted large Danish registry study demonstrated that having any mental disorder significantly predisposes to the development of a subsequent disorder, at least for disorders warranting psychiatric care. ${ }^{[4]}$ Although this may have contributed to the low rates of severe depression and anxiety found by Lu et al. (2020) ${ }^{[12]}$ who excluded staff members with a previous mental disorder, their use of detailed rating scales may also be a factor.

Thirdly, comorbidity between mental disorders was inadequately addressed to permit accurate interpretation of the prevalence rates. In SA, a 2004 nationally representative mental health survey using a diagnostic interview ${ }^{[45]}$ identified a depressive, anxious, PTS and/ or substance use disorder in $16.5 \%$ of respondents. Of these, $30 \%$ met criteria for two or more disorders, with greater disorder severity associated with higher levels of comorbidity. In addition, conditions such as insomnia and somatisation may also occur as symptoms of other mental disorders. The cluster analysis by Kang et al. ${ }^{[17]}$ may therefore be the most meaningful of the studies included in our review for planning of HCW support. Interestingly, the prevalence of severe mental disturbance (6.2\%) found by Kang et al. is similar to the rates of severe depression (6.2\%) and severe anxiety (5.3\%) found by Lai et al., ${ }^{[11]}$ where the same individual HCWs may be represented in each condition.

Lastly, no study evaluated the impact of mental health conditions on HCW functioning in the workplace. However, executive or cognitive dysfunction, with social or occupational impairment, are core features of almost all mental disorders. ${ }^{[46]}$ Furthermore, although burnout, stress, moral injury and fear are not disorders but normal reactions to taxing circumstances, they impact negatively on workplace function, and they may predispose to or be comorbid with mental disorders.

\section{Risk factors}

Although infectious disease exposure and workplace stressors are prominent risk factors for mental health conditions, working at a tertiary hospital may be protective. ${ }^{[11-13]}$ Health system influence is also evident in studies reporting on stress related to resource constraints. ${ }^{[12,29,31,35]}$ A possibility is that tertiary hospitals are better equipped in respect of infection control, clinical infrastructure and skilled staff than secondary-level facilities, which may be pressured to provide services beyond their capacity during an outbreak. Poor infrastructure may cause over-exposure of HCWs to high caseloads and negative patient outcomes, with feelings of incompetence, moral injury and emotional exhaustion. The effect of resource constraints has important implications for HCWs in sub-Saharan Africa, where weak health infrastructure and poor healthcare outcomes are well documented under normal healthcare service conditions. ${ }^{[47]}$

Nurses were noted to be at high risk of work-related stress and mental health conditions, whether frontline or not. ${ }^{[8,10,11,18,34]}$ While this may be related to a preponderance of nurses in study samples, it may reflect a convergence of multiple risk factors, including poor control over working conditions, poor management support, being female, and having family responsibilities. Similar organisational dynamics, including the influence of gender, are discussed in a recent scoping review highlighting severe nursing shortages in low- and middle-income countries. ${ }^{[48]}$ While such shortages contribute to increased work demands and stress, only one study in our review examined the impact of an outbreak on turnover intention among nurses, ${ }^{[15]}$ and none evaluated nursing workplace planning.

The mental health risk to doctors and to men may be underrecognised, affected by sampling strategies, response rates and the conditions investigated. As well as not evaluating non-responders, surveys measuring specific conditions may miss other manifestations of distress possibly more common among men or doctors. Of note, burnout, examined in only one small sample of $\mathrm{HCWs},{ }^{[10]}$ was greater among doctors compared with nurses. Additionally, Dubale et al..$^{[42]}$ found burnout to be highly prevalent among doctors, nurses and other HCWs in sub-Saharan Africa. With no mention of gender, they list heavy workloads, poor working conditions and staff shortages as risk factors for burnout, while management support was found to be protective.

Finally, it is evident that high rates of mental health conditions, burnout and stress among frontline and non-frontline HCWs may be anticipated during the COVID-19 response in Africa. While nurses, women, and those with poor social support are at particular risk, other HCWs are also affected. The lack of primary care practitioner representation in studies implies that this cadre of HCW is at risk of neglect.

\section{Interventions}

The interventions we identified were all proposed in opinion pieces or in discussion of study results, suggesting that they are open to interpretation and selective application according to local conditions. However, two main principles emerge: $(i)$ the institution and management are the key role players; and (ii) accessible, appropriate psychological support is needed.

These principles are consistent with WHO guidelines for HCWs in public health emergencies ${ }^{[3]}$ and for ensuring mental health in the workplace. ${ }^{[4]}$ Fundamental institutional responsibilities include provision of adequate health infrastructure, infection prevention and control, workforce staffing appropriate for the caseload, and flexible working hours. However, these may not be readily implementable in sub-Saharan Africa. As noted by Kruk et al. (2018), ${ }^{[47]}$ health system quality deficits are deeply entrenched, requiring intensive reform involving governments and civil society. Furthermore, the severe nursing shortages ${ }^{[48]}$ and already high levels of burnout ${ }^{[42]}$ may preclude selective HCW deployment.

Nevertheless, certain strategies identified in this review may be implementable in resource-constrained settings, some of which are listed in Table 4. Our suggestions are consistent with findings by Gray et al. (2019) ${ }^{[5]}$ on organisation-level interventions to promote mental health among HCWs, drawn mainly from studies in high- 
Table 4. Suggested interventions

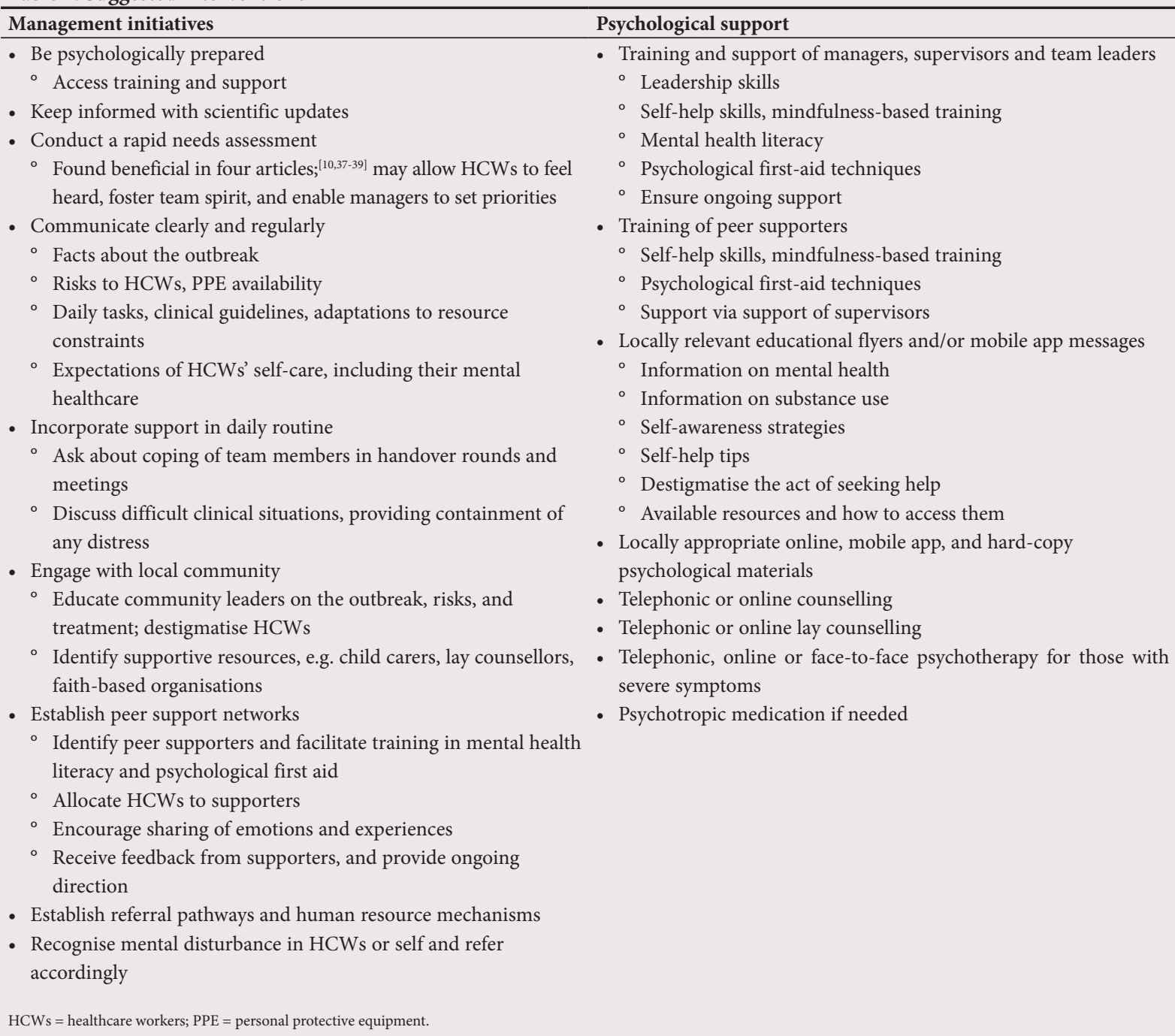

income countries. While educational leaflets are possibly the simplest to implement, skilled leadership and effective communication are pivotal. Psychological support and training of managers is therefore prioritised, with individual care reserved for HCWs with severe symptoms. Use of non-governmental and volunteer organisations could help overcome shortages of mental health professionals.

\section{Study limitations}

Several limitations of this article should be noted. Firstly, the rapid nature of the review in response to a local request precluded an a priori protocol as well as duplicate study selection and data extraction. Secondly, using the systematic review by Brooks et al.$^{[8]}$ as a starting point may have excluded other relevant studies. Thirdly, the inclusion of small, informal studies such as rapid needs assessments as research may overrate their findings.

\section{Conclusions}

This review found that HCWs experience high levels of mental health disturbance during infectious disease outbreaks. Further research on mental health of HCWs in Africa, particularly those in the primary care setting and with workplace-related outcomes, is urgently needed. While no intervention effectiveness studies were identified, several strategies that leadership may undertake were noted, and implementation should not be delayed.

Dedication. This publication is dedicated to the late Associate Professor Bernard Janse van Rensburg, who, at the time of his death in April 2020, was working with a number of mental health professional societies towards providing psychological support to healthcare workers across South Africa during the COVID-19 outbreak.

\section{Declaration. None.}

Acknowledgements. The authors would like to acknowledge Ms Tsholofelo Adelekan and Dr Bridget Ikalafeng of the Policy, Planning, Research and Health Information Evaluation and Monitoring Directorate and Dr Sipho Senabe of the Occupational Health and Safety Department in the Gauteng Department of Health for providing strategic direction and support for this research.

Author contributions. LJR conceptualised and designed the work, conducted the data extraction, analysis and interpretation, and drafted the manuscript. OJ, IM and HS assisted with the preliminary screening of the 
literature, data analysis and interpretation, as well as review and editing of the manuscript. OJ brought the authors together as a task team and initiated the project.

\section{Funding. None.}

\section{Conflicts of interest. None.}

1. Abd El-Aziz TM, Stockand JD. Recent progress and challenges in drug development against COVID-19 coronavirus (SARS-CoV-2) - an update on the status. Infect Genet Evol 2020;83:104327. https://doi.org/10.1016/j.meegid.2020.104327

2. World Health Organization. WHO Director-General's opening remarks at the media briefing on COVID-19 - 11 March 2020. 11 March 2020. https://www.who.int/dg/speeches/detail/ who-director-general-s-opening-remarks-at-the-media-briefing-on-covid-19---11-march-2020 (accessed 6 May 2020)

3. World Health Organization and International Labour Organization. Occupational Safety and Health in Public Health Emergencies: A Manual for Protecting Health Workers and Responders. Health in Public Health Emergencies: A Manual for Protecting Health Workers and Responders.
Geneva, 2018. https://www.who.int/occupational_health/publications/safety-health-public-healthGeneva, 2018. https://www.who.int/occupt
emergencies/en/ (accessed 6 May 2020).

emergencies/en/ (accessed 6 May 2020).
4. World Health Organization. Mental health in the workplace: Information sheet. May 2019. https:// 4. World Health Organization. Mental health in the workplace: Information shet
www.who.int/mental_health/in_the_workplace/en/(accessed 6 May 2020).

5. Gray P, Senabe S, Naicker N, Kgalamono S, Yassi A, Spiegel JM. Workplace-based organizational interventions promoting mental health and happiness among healthcare workers: A realist review. Int J Environ Res Public Health 2019;16(22):4396. https://doi.org/10.3390/ijerph16224396

6. Pfefferbaum B, North CS. Mental health and the Covid-19 pandemic. N Engl J Med 2020;383:510512. https://doi.org/10.1056/NEJMp2008017

7. Brooks SK, Webster RK, Smith LE, et al. The psychological impact of quarantine and how to reduce it: Rapid review of the evidence. Lancet 2020;395(10227):912-920. https://doi.org/10.1016/s01406736(20)30460-8

8. Brooks SK, Dunn R, Amlot R, Rubin GJ, Greenberg N. A systematic, thematic review of social and occupational factors associated with psychological outcomes in healthcare employees during and occupational factors associated with psychological outcomes in healthcare employees during
an infectious disease outbreak. J Occup Environ Med 2018;60(3):248-257. https://doi.org/10.1097/ an infectious disease out

9. Tricco AC, Lillie E, Zarin W, et al. PRISMA Extension for Scoping Reviews (PRISMA-ScR): Checklist and explanation. Ann Intern Med 2018;169(7):467-473. https://doi.org/10.7326/M18-0850

10. Cao J, Wei J, Zhu H, et al. A study of basic needs and psychological wellbeing of medical workers in the fever clinic of a tertiary general hospital in Beijing during the COVID-19 outbreak. Psychothe Psychosom 2020;89:252-254. https://doi.org/10.1159/000507453

11. Lai J, Ma S, Wang Y, et al. Factors associated with mental health outcomes among health care workers exposed to coronavirus disease 2019. JAMA Netw Open 2020;3(3):e203976. https://doi.org/10.1001 jamanetworkopen.2020.3976

12. Lu W, Wang H, Lin Y, Li L. Psychological status of medical workforce during the COVID-19 pandemic: A cross-sectional study. Psychiatry Res 2020;288:112936. https://doi.org/10.1016/j. psychres.2020.112936

13. Tan BYQ, Chew NWS, Lee GKH, et al. Psychological impact of the COVID-19 pandemic on health care workers in Singapore. Ann Intern Med 2020;173(4):317-320. https://doi.org/10.7326/M20-1083

14. Zhang WR, Wang K, Yin L, et al. Mental health and psychosocial problems of medical health workers during the COVID-19 epidemic in China. Psychother Psychosom 2020;89:242-250. https:// workers during the COVID-
doi.org/10.1159/000507639

15. Jung H, Jung SY, Lee MH, Kim MS. Assessing the presence of post-traumatic stress and turnover intention among nurses post-Middle East respiratory syndrome outbreak: The
turs turnover intention among nurses post-Middle East respiratory syndrome outbreak: The
importance of supervisor support. Workplace Health Saf 2019;68(7):337-345. https://doi. org/10.1177/216507991989769

16. Lee SM, Kang WS, Cho AR, Kim T, Park JK. Psychological impact of the 2015 MERS outbreak on hospital workers and quarantined hemodialysis patients. Compr Psychiatry 2018;87:123-127. https://doi.org/10.1016/j.comppsych.2018.10.003

17. Kang L, Ma S, Chen M, et al. Impact on mental health and perceptions of psychological care among medical and nursing staff in Wuhan during the 2019 novel coronavirus disease outbreak: A crosssectional study. Brain Behav Immun 2020;87:11-17. https://doi.org/10.1016/j.bbi.2020.03.028

18. Mo Y, Deng L, Zhang L, et al. Work stress among Chinese nurses to support Wuhan for fighting against the COVID-19 epidemic. J Nurs Manag 2020;28(5):1002-1009. https://doi.org/10.1111/ jonm. 13014

19. Li Z, Ge J, Yang M, et al. Vicarious traumatization in the general public, members, and non-member of medical teams aiding in COVID-19 control. Brain Behav Immun 2020;88:916-919. https://doi. org/10.1016/j.bbi.2020.03.007

20. Greenberg N, Docherty M, Gnanapragasam S, Wessely S. Managing mental health challenges faced by healthcare workers during covid-19 pandemic. BMJ 2020;368:m1211. https://doi.org/10.1136/ by healthcare wi.m1211

21. Walton M, Murray E, Christian MD. Mental health care for medical staff and affiliated healthcare workers during the COVID-19 pandemic. Eur Heart J Acute Cardiovasc Care 2020;9(3):241-247. https://doi.org/10.1177/2048872620922795

2. Ulrich CM. Ebola is causing moral distress among African healthcare workers. BMJ 2014;349:g6672. https://doi.org/10.1136/bmj.g6672

23. Ho CS, Chee CY, Ho RC. Mental health strategies to combat the psychological impact of COVID-19 beyond paranoia and panic. Ann Acad Med Singapore 2020;49(3):155-160.
24. Wu AW, Connors C, Everly GS Jr. COVID-19: Peer support and crisis communication strategies to promote institutional resilience. Ann Intern Med 2020;172(12):822-823. https://doi.org/10.7326/ M20-1236

25. Kang L, Li Y, Hu S, et al. The mental health of medical workers in Wuhan, China dealing with the 2019 novel coronavirus. Lancet Psychiatry 2020;7(3):E14. https://doi.org/10.1016/s2215$0366(20) 30047-x$

26. Wu P, Fang Y, Guan Z, et al. The psychological impact of the SARS epidemic on hospital employees in China: Exposure, risk perception, and altruistic acceptance of risk. Can J Psychiatry 2009;54(5):302311. https://doi.org/10.1177/070674370905400504

27. Greenberg N, Wessely S, Wykes T. Potential mental health consequences for workers in the Ebola regions of West Africa - a lesson for all challenging environments. J Ment Health 2015;24(1):1-3, https://doi.org/10.3109/09638237.2014.1000676

28. Huang J, Liu F, Teng Z, et al. Care for the psychological status of frontline medical staff fighting against COVID-19. Clin Infect Dis 2020 (epub 21 April 2020). https://doi.org/10.1093/cid/ciaa385

29. Raven J, Wurie H, Witter S. Health workers' experiences of coping with the Ebola epidemic in Sierra Leone’s health system: A qualitative study. BMC Health Serv Res 2018;18(1):251. https://doi, org/10.1186/s12913-018-3072-3

30. Alikhani R, Salimi A, Hormati A, Aminnejad R. Mental health advice for frontline healthcare providers caring for patients with COVID-19. Can J Anaesth 2020;67:1068-1069. https://doi. org /10.1007/s12630-020-01650-3

31. Chen Q, Liang M, Li Y, et al. Mental health care for medical staff in China during the COVID-19 outbreak. Lancet Psychiatry 2020;7(4):e15-e16. https://doi.org/10.1016/s2215-0366(20)30078-x

32. Xiao H, Zhang Y, Kong D, Li S, Yang N. The effects of social support on sleep quality of medical staff treating patients with coronavirus disease 2019 (COVID-19) in January and February 2020 in China. Med Sci Monit 2020;26:e923549. https://doi.org/10.12659/MSM.923549

33. Khalid I, Khalid TJ, Qabajah MR, Barnard AG, Qushmaq IA. Healthcare workers emotions, perceived stressors and coping strategies during a MERS-CoV outbreak. Clin Med Res 2016;14(1):714. https://doi.org/10.3121/cmr.2016.1303

34. Park JS, Lee EH, Park NR, Choi YH. Mental health of nurses working at a government-designated hospital during a MERS-CoV outbreak: A cross-sectional study. Arch Psychiatr Nurs 2018;32(1):2-6. https://doi.org/10.1016/j.apnu.2017.09.006

35. Shanafelt T, Ripp J, Trockel M. Understanding and addressing sources of anxiety among health care professionals during the COVID-19 pandemic. JAMA 2020;323(21):2133-2134. https://doi. org/10.1001/jama.2020.5893

36. Ripp J, Peccoralo L, Charney D. Attending to the emotional well-being of the health care workforce in a New York City health system during the COVID-19 pandemic. Acad Med 2020;95(8):11361139. https://doi.org/10.1097/ACM.0000000000003414

37. Schreiber M, Cates DS, Formanski S, King M. Maximizing the resilience of healthcare workers in multi-hazard events: Lessons from the 2014 - 2015 Ebola response in Africa. Mil Med 2019;184(Suppl 1):114-120. https://doi.org/:10.1093/milmed/usy400

38. Wu PE, Styra R, Gold WL. Mitigating the psychological effects of COVID-19 on health care workers. Can Med Assoc J 2020;192(17):E459-E460. https://doi.org/10.1503/cmaj.200519

39. Yin X, Zeng L. A study on the psychological needs of nurses caring for patients with coronavirus disease 2019 from the perspective of the existence, relatedness, and growth theory. Int J Nurs Sci 2020;7(2):157-160. https://doi.org/10.1016/j.ijnss.2020.04.002

40. Rossouw L, Seedat S, Emsley RA, Hagenmeister D. The prevalence of burnout and depression in medical doctors working in the Cape Town Metropolitan Municipality community healthcare clinics and district hospitals of the Provincial Government of the Western Cape: A cross-sectional study. S Afr Fam Pract 2013;55(6):567-573. https://doi.org/10.1080/20786204.2013.10874418

41. Govender I, Mutunzi E, Okonta HI. Stress among medical doctors working in public hospitals of the Ngaka Modiri Molema district (Mafikeng health region), North West province, South Africa. S Afr J Psychiatry 2012;18(2):42-46. https://doi.org/10.4102/sajpsychiatry.v18i2.337

42. Dubale BW, Friedman LE, Chemali Z, et al. Systematic review of burnout among healthcare providers in sub-Saharan Africa. BMC Public Health 2019;19(1). https://doi.org/10.1186/s12889019-7566-7

43. World Health Organization. Burn-out an 'occupational phenomenon': International Classification of Diseases. 28 May 2019. https://www.who.int/mental_health/evidence/burn-out/en/ (accessed 20 May 2020).

44. Plana-Ripoll O, Pedersen CB, Holtz Y, et al. Exploring comorbidity within mental disorders among a Danish national population. JAMA Psychiatry 2019;76(3):259-270. https://doi.org/10.1001/ jamapsychiatry.2018.3658

45. Williams DR, Herman A, Stein DJ, et al. Twelve-month mental disorders in South Africa: Prevalence, service use and demographic correlates in the population-based South African Stress and Health Study. Psychol Med 2008;38(2):211-220. https://doi.org/10.1017/S0033291707001420

46. American Psychiatric Association DSM-5 Task Force. Diagnostic and Statistical Manual of Mental Disorders: DSM-5. 5th ed. Washington, DC: American Psychiatric Association, 2013.

47. Kruk ME, Gage AD, Arsenault C, et al. High-quality health systems in the Sustainable Development Goals era: Time for a revolution. Lancet Glob Health 2018;6(11):e1196-e1252. https://doi. org/10.1016/\$2214-109X(18)30386-3

48. Drennan VM, Ross F. Global nurse shortages - the facts, the impact and action for change. Br Med Bull 2019;130(1):25-37. https://doi.org/10.1093/bmb/ldz014

Accepted 25 August 2020. 